Годишник Телекомуникации 2019, том 6, с. 75-82

Yearbook Telecommunications 2019, vol. 6, p. 75-82

eISSN 2534-854X

https://telecommunications.nbu.bg/bg/godishnik-telekomunikacii

DOI: https://doi.org/10.33919/YTelecomm.19.6.8

АНАЛИЗ И ОЦЕНКА НА РИСКА ОТ РИСКОВИ ТЕХНИЧЕСКИ СИСТЕМИ ПРИ РАЗРАБОТВАНЕТО НА МЕТОДИКА ЗА ОБУЧЕНИЕТО НА СТУДЕНТИ ЧРЕЗ ТРАНСФОРМИРАНЕ НА СТРУКТУРИ

\author{
Цветелина Симеонова
}

\title{
ANALYSIS AND RISK ASSESSMENT OF RISK TECHNICAL SYSTEMS IN THE DEVELOPMENT OF THE METHODOLOGY FOR THE STUDENTS TRAINING BY TRANSFORMING STRUCTURES
}

\author{
Tsvetelina Simeonova
}

\begin{abstract}
Резюме: Да се разработи методика за анализ на риска чрез трансформиране на структури. Предложена е методика за анализ на риска чрез трансформиране на структури, базирана на съществуващи връзки между описание на системата чрез блокова диаграма, дърво на отказите и дърво на събитията. Анализът на риска от рискови технически системи чрез дърво на отказите и дърво на събитията дава еднозначна връзка между параметри на елементи и параметри на еквивалентни елементи, описващи система или подсистема.

Показан е пример за анализ на риска от чрез трансформиране на структури, съгласно предложената методика, приложим за определяне на риска, включващ трансформации между блокова диаграма и дърво на отказите и дърво на събитията, както и варианти за определяне на риска по тях (при приета стойност на вредите), съгласно направени приемания.

Разработена е методика и са показани примери за анализ на риска чрез трансформиране на структури.

Ключови думи: надеждност, риск, трансформация на структури, надеждностна блокова диаграма, дърво на отказите, дърво на събитията.
\end{abstract}

\begin{abstract}
The purpose of this article is to develop a methodology for risk analysis by transforming structures. A methodology for risk analysis is proposed by transforming structures based on existing links between the system description through a block diagram, a fault tree, and an event tree. Risk analysis of risk techniques by fault tree and event tree provides an unambiguous relationship between element parameters and parity of equivalent elements describing a system or a subsystem.

An example of risk analysis by transforming structures is presented, according to the proposed methodology applicable to risk identification, including transformations between block diagram and fault tree and event tree, as well as variants to determine their risk (at accepted value of damages), according to acceptances made.

A methodology has been developed and examples of risk analysis by transformation of structures are shown.
\end{abstract}

Keywords: reliability, risk, structure transformation, reliability block diagram, fault tree, event tree.

\section{1. ВЪВЕДЕНИЕ}

Адекватното усвояване на теорията и методите за изследване на надеждността е основа за правилното и безгрешно прилагане от студентите на съответните еквивалентни трансформации, между надеждностна блокова диаграма, дърво на отказите и дърво на събитията и използването им като методи за определяне на вероятността за поява на нежелано сьбитие, използвана при определянето на риска $[1,2,3,4,5]$.

Структурните методи представят изследваната система във формата на логически структурно-функционални схеми (чрез които се изгражда модел). 


\section{АНАЛИЗ И ОЦЕНКА НА РИСКА ОТ РИСКОВИ ТЕХНИЧЕСКИ СИСТЕМИ ПРИ \\ РАЗРАБОТВАНЕТО НА МЕТОДИКА ЗА ОБУЧЕНИЕТО НА СТУ ДЕНТИ ЧРЕЗ \\ ТРАНСФОРМИРАНЕ НА СТРУКТУРИ}

\section{ЦВЕТЕЛИНА СИМЕОНОВА}

Предложена е разработена методика приложима както при надеждностни изследвания, така и при определяне и анализ на риска, при възникване на опасно събитие чрез трансформиране на структури. При изследване на риска от техническа система, за определяне на вероятността за поява на опасно събитие, може да се използват различни логически модели - или надеждностна блокова диаграма или метода дърво на отказите или метода дърво на събитията. Трите метода са свързани и може да бъде използван всеки от тях, при наличие на описание по другия, а кой метод ще бъде предпочетен зависи от конкретното приложение, с отчитане на предимствата и недостатъците.

Моделът може да бъде от различно ниво на детайлност в зависимост от сложността и необходимостта от подробен анализ на изследваната система, като за получаване на вероятността за реализиране на опасно събитие се използват изчислителни методи за определяне най-често на надеждностни показатели. Целият процес на изграждане на модел от който определяме риска на системата може да се представи като последователност от отделни задачи и дейности по реализирането им.

\section{1. Надеждностна блокова диаграма}

Блоковите структурни схеми по надеждност (например показаната на фиг. 1) представят въздействието, което оказват отказите в елементите на дадена система върху нейната обща надеждност, като се разделят на последователни, паралелни, смесени и сложни структури $[1,2]$.

В повечето случаи функционирането на системата се описва с помощта на по-сложни техники за моделиране на надеждността от надеждностните блокови схеми, такива са методите "дърво на отказите" и "дърво на събитията". При тях връзките и зависимостите между отделните елементи на системата се представят във формата на дървовидна структура, което улеснява анализа.

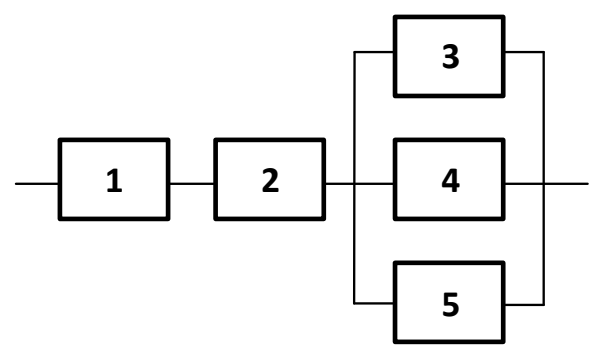

Фиг. 1. Примерна блокова структура.

\section{2. Дьрво на отказите}

Дьрвото на отказите е графично представяне на събитията в дървовидна структура [3]. Методът е широко разпространен и се основава на Булевата алгебра и вероятностната логика. Събитие в дървото на отказите може да бъде: отказ на елемент, човешки грешки, дестабилизиращ фактор и т.н. Събитията се разделят на два типа: входни (input events) и изходни (output events).

За построяване на дървото на отказите се задава нежеланото изходно събитие (върхов отказ - "top event") и се търсят причините, които могат да го породят. Причините за поява на нежеланото събитие на изхода се представят графично в логическа дървовидна йерархична структура (отгоре-надолу), в която събитията се моделират посредством символи, а логически елементи отразяват отношението между тях. 
Годишник Телекомуникации 2019, том 6, с. 75-82

Yearbook Telecommunications 2019, vol. 6, p. 75-82

\section{3. Дърво на събитията}

Диаграмата на дървото на събитията $[4,5]$ моделира всички възможни пьтища от началното събитие. Иницииращото събитие започва от лявата страна като хоризонтална линия, която се разклонява вертикално. В края на вертикалния клон се изчертават хоризонтални линии от горната и долната част, представляващи успеха или неуспеха на събитието, като се обозначава с етикет, който описва пътя. Този процес продължава до достигане на крайното състояние (крайните състояния се класифицират в групи, които могат да бъдат "успех" или "тежест на последствията"). Когато диаграмата на дървото на събитията е достигнала крайното състояние за всички пътища, се записва уравнението на вероятност за изход.

\section{2. ЕКВИВАЛЕНТНИ ПРЕОБРАЗУВАНИЯ МЕЖДУ НАДЕЖДНОСТНА БЛОКОВА ДИАГРАМА, ДЪРВО НА ОТКАЗИТЕ И ДЪРВО НА СЬБИТИЯТА $[6,7,8,9,10]$}

В този раздел се запознаваме с техниките и приложението на трансформацията (преобразуването) между надеждностна блокова диаграма (Reliability' block diagram), дърво на отказите (Fault tree) и дърво на събитията (Event tree), т.е. имаме:

1. трансформация от надеждностна блокова диаграма към дърво на отказите;

2. трансформация от надеждностна блокова диаграма към дърво на събития;

3. трансформация от дърво на отказите към дърво на събитията;

4. трансформация от дърво на отказите към надеждностна блокова диаграма;

5. трансформация от дърво на събитията към надеждностна блокова диаграма;

6. определяне от надеждностната блокова диаграма на: сеченията (cut sets) и набора от пьтища (path sets).

Надеждностна блокова диаграма, дърво на отказите и дърво на събитията са символични логически модели. По принцип дефинираме области (домейни) на успех и неуспех (отказ). $\mathrm{B}$ домейна за откази се генерират дървета на откази, в домейна за успех се генерират надеждностни блокови диаграми, а в домейна за успех и откази се генерират дървета на събития. По такъв начин са допустими трансформации между символичните логически модели, отчитайки техните домейни [6].

Трансформациите обикновено се изпълняват в етапа на проектиране и разработване, но може също така да бъдат изпълнени във фазата на дефиниране на концепцията.

Описаните трансформации позволяват да се използват, за всеки конкретен случай, съответните предимства на всеки от символичните логически модели, а именно:

- Надеждностната блокова диаграма предлага опростен метод за представяне на системната логика.

- Дьрветата на отказите предлагат цялостен качествен или количествен анализ.

- Дърветата на събитията позволяват да се оцени системата както в областите за успех, така и в областите на отказ.

По принцип всеки отказ на система става по няколко начина [10]: - различни пътища към най-високото събитие (отказ на система).

- Cut set ${ }^{1}$ - - основни събития, които водят до най-високото събитие (отказ на система);

\footnotetext{
${ }^{1}$ Cut Set сечение на блоковата диаграма, включващо елементи, отказът на които води до отказ на системата
} 


\section{АНАЛИЗ И ОЦЕНКА НА РИСКА ОТ РИСКОВИ ТЕХНИЧЕСКИ СИСТЕМИ ПРИ \\ РАЗРАБОТВАНЕТО НА МЕТОДИКА ЗА ОБУЧЕНИЕТО НА СТУ ДЕНТИ ЧРЕЗ \\ ТРАНСФОРМИРАНЕ НА СТРУКТУРИ}

ЦВЕТЕЛИНА СИМЕОНОВА

сечение от всички възможни набори на откази на компоненти, водещи до отказ;

- Minimal cut set²: - премахването на основно събитие избягва отказ; минимално сечение набор на откази на компоненти (последователно и/или паралелно свързани), съобразно всички възможни пътища, отказ на всеки от които или едновременен отказ на паралелно свързаните, води до финално събитие (отказ на системата);

- Path set ${ }^{3}$ - Набор път (сумарно всички възможни пътища (съставени от последователни и паралелни компоненти), които избягват финалното събитие (отказ на системата), поради което са успешни):

- основни събития, които избягват най-високото събитие (отказ);

- списьк на компонентите, които гарантират безопасността.

Въз основа на стойностите, които се получават може да се направят изводи за минималните сечения на дървото на отказите [11].

Качествената и количествената оценка се основават на минималните сечения на дървото на отказите. Най-малката подгрупа на всяко сечение, която предизвиква отказ на системата, се нарича минимално сечение. Може да се даде следното определение: минимално сечение е такава група, която се състои от най-малко на брой елементи, чиито едновременен отказ ще предизвика отказ на системата.

\section{Трансформации}

Представени са процедурите за трансформиране на надеждностна блокова диаграма, дърво на отказите и на дърво на събитията в някой от другите два логически модела [6].

\section{Трансформация на Дьрво на отказите в надеждностна блокова диаграма}

Надеждностната блокова диаграма представя функциите на системните компоненти, които, ако тези функции преобладават, дават успех вместо финалното събитие (отказ) по метода Дьрво на отказите. Използвайки тази особеност Дьрво на отказите може да се трансформира в надеждностна блокова диаграма.

Трансформация на надеждностна блокова диаграма и на Дьрво на отказите в Дърво на събитията

Дьрвото на събитията представя path sets (набори от пътища) в клоновете на успех (без откази) на дървото и всички cut sets (сечения) в клоновете с откази на дървото. Следователно, ако path sets и cut sets (наборите на пътя и наборите на системата) са известни, тогава може да се конструира дърво на събития; те могат да бъдат определени например от надеждностна блок диаграма.

Определянето на минималните сечения (Cut set) и пътищата (Path set) от надеждностна блокова диаграма от фиг. 1 е дадено на фиг. 2.

За големи комплексни дървета на отказите, сеченията (cut sets) и наборите от пьтища (path sets) се получават с помощта на алгоритъма на метода MOCUS (Method of Obtaining Cut Sets). Методът се разглежда като един от традиционните алгоритми за намаляване големината на дърветата на отказите и др.

Методът възприема подход "отгоре-надолу", като се започва от корена (финално събитие) и се продължава като се декомпозира всеки логически елемент на по-високо ниво, докато

\footnotetext{
${ }^{2}$ Minimal Cut Set минимално сечение на блоковата диаграма, включващо минимален брой елементи отказът на които води до отказ, използва се при анализ по дърво на отказите.

${ }^{3}$ Path Set набор път на блоковата диаграма, включващ неотказали елементи, или сечение на път водещо до отсъствие на отказ, използва се при анализ по дърво на събитията.
} 
Годишник Телекомуникации 2019, том 6, с. 75-82

Yearbook Telecommunications 2019, vol. 6, p. 75-82

се определят само основните събития.

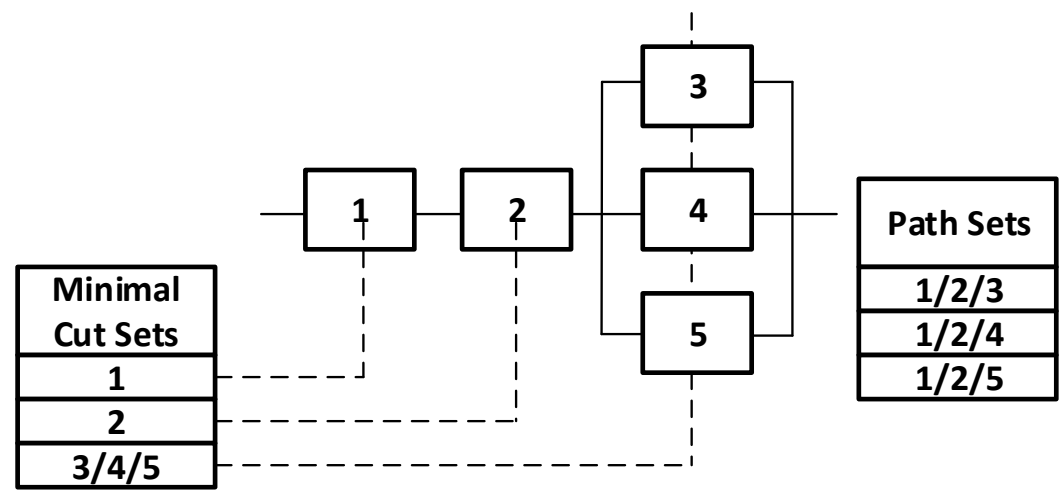

Фиг. 2. Минимални сечения и пьтища.

Трансформиране на надеждностна блокова диаграма от фиг. 1 и фиг. 2 в дърво на събитията е показано на фиг. 3. За да се трансформира дърво на отказите в дърво на събитията, първо се трансформира дървото на отказите в надеждностна блокова диаграма.

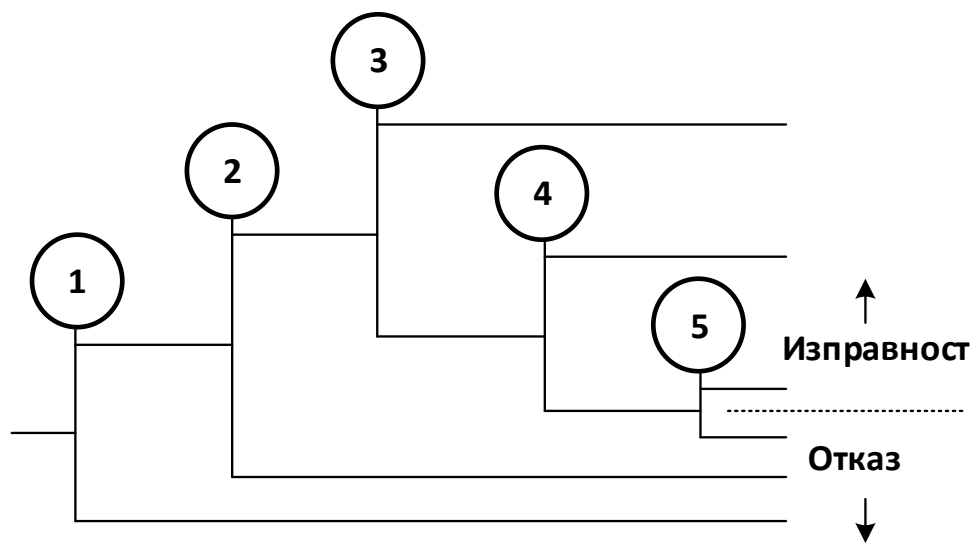

Фиг. 3. Трансформиране на надеждностна блокова диаграма в дърво на събитията.

\section{Трансформация на надеждностна блокова диаграма в дърво на отказите}

Дървото на отказите представя системни функции, които, ако откажат, водят до отказ на финалното събитие, а не събитие за успех (отсъствие на отказ), към което води съответната надеждностна блокова диаграма. Последователно свързаните възли на надеждностната блокова диаграма означават логически елемент OR под финалното събитие на дървото на отказите. Паралелните свързвания в надеждностната блокова диаграма означават логически елемент AND за функциите на резервния компонент в дървото на отказите.

Следователно, надеждностната блокова диаграма от фиг. 1 може да се преобразува в дърво на отказите, както е показано на фиг. 4. 


\section{АНАЛИЗ И ОЦЕНКА НА РИСКА ОТ РИСКОВИ ТЕХНИЧЕСКИ СИСТЕМИ ПРИ \\ РАЗРАБОТВАНЕТО НА МЕТОДИКА ЗА ОБУЧЕНИЕТО НА СТУ ДЕНТИ ЧРЕЗ \\ ТРАНСФОРМИРАНЕ НА СТРУКТУРИ}

ЦВЕТЕЛИНА СИМЕОНОВА

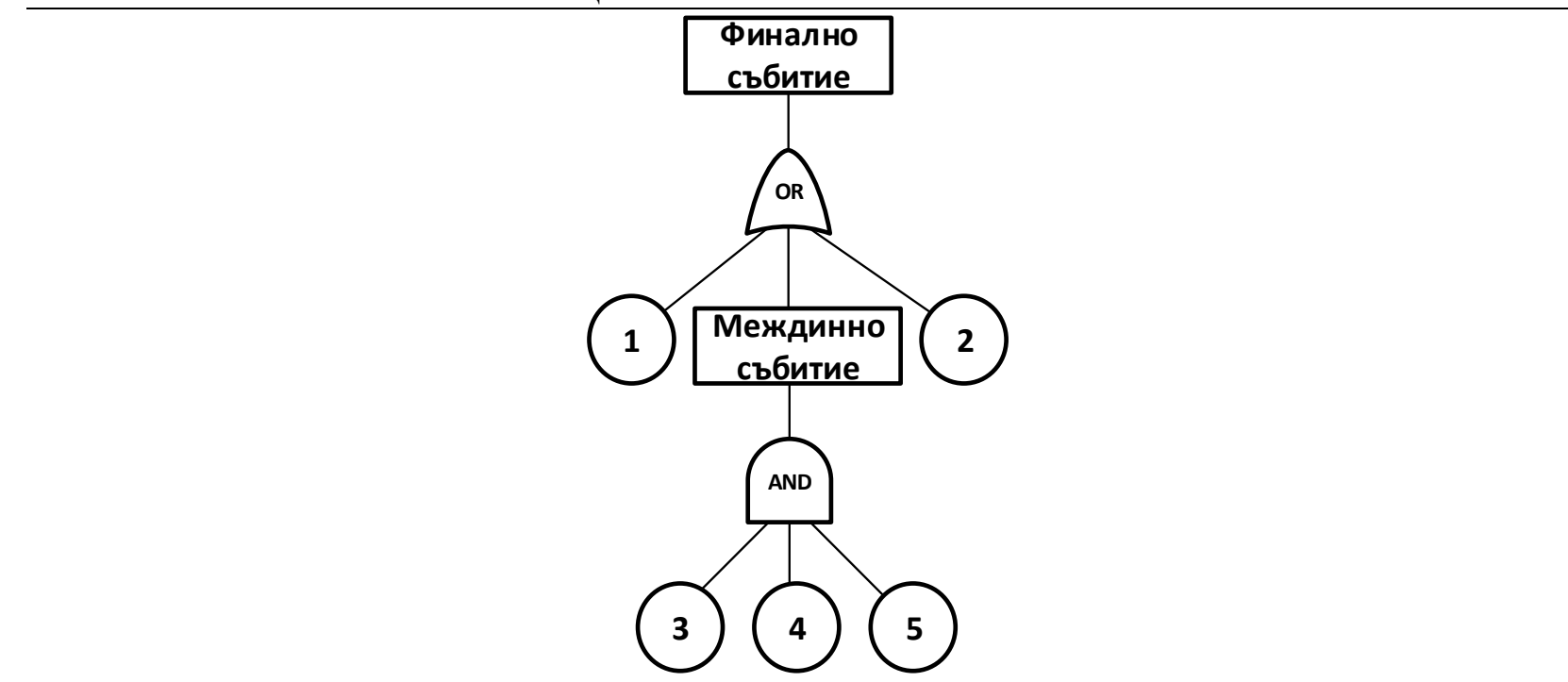

Фиг. 4. Трансформиране на надеждностната блокова диаграма в дърво на отказите

Дьрвото на събитията представя набори от пътища (path sets) в клоновете за успех на дървото и всички сечения (cut sets) в отказалите клони на дървото. За да трансформирате дървото на събитията в надеждностната блокова диаграма, обърнете процеса, илюстриран на фигура 3. След формирането на надеждностната блокова диаграма може да се преобразува в дърво на отказите.

По отношение на трансформирането на дърво на събитията в дърво на отказите е важно да се помни, че дървото на събитията се занимава както с успеха (липса на отказ), така и с неуспеха (отказ).

По този начин, някои от стойностите на вероятностите, използвани в дървото на събитията, са вероятности за успех $\left(\mathrm{Ps}=1-\mathrm{P}_{\mathrm{F}}\right)$. Тъй като дървото на събитията започва с иницииращо събитие и може да доведе до крайни събития чрез многобройни сценарии, дървото на отказите, асоциирано с дърво на събития, ще се състои от групи т.нар. "храсти на отказите", по една за всеки път до отказ (от набора), и те са свързани към финалното събитие през логически елемент ИЛИ (OR).

Предимства: Подходът за трансформиране на структури позволява да се преодолеят слабостите на техниката за анализ чрез трансформиране на системен модел в еквивалентен логически модел (който е подходящ за друга техника за анализ). Например, сложна система, която може да бъде трудна за моделиране директно чрез дърво на отказите, може лесно да бъде моделирана с надеждностна блокова диаграма. След това надеждностната блокова диаграма може да се трансформира в дърво на отказите и може да се извърши подробен количествен (или псевдо-количествен) анализ.

Ограничения: Не се получава нова информация относно системата и моделите са толкова добри, колкото и моделите, които се трансформират.

За големи системи, определянето на сеченията (cut sets) и наборите от пътища (path sets), които са необходими за да се извършат трансформации, е възможно да изискват много човеко-часове и/или големи компютърни ресурси.

\section{3. ИЗСЛЕДВАНЕ НА РИСКА ЧРЕЗ ТРАНСФОРМИРАНЕ НА СТРУКТУРИ.}

Разработена е методика за определяне и анализ на риска за възникване на опасно събитие (опасен отказ на техническа система) чрез трансформиране на структури с използване на методите "дърво на отказите FT (Fault Tree)" и "дърво на събитията ET (Event Tree)". 
Годишник Телекомуникации 2019, том 6, с. 75-82

Yearbook Telecommunications 2019, vol. 6, p. 75-82

Показан е пример за задание на надеждностна блокова структура от фиг. 1, на която се определя риска чрез метода "дърво на отказите" и метода "дърво на събитията", като зададената структура от фиг. 1 се трансформира до съответните структури - на ЕТ (фиг. 3) и на FT (фиг. 4).

Приема се, че всички елементи на зададените две блокови структури са с еднакви параметри за вероятност за отказ на компонент.

Да се определи риска чрез определяне вероятността за поява на:

- финалното събитие при FT;

- $\quad$ крайните събития (следствията) при ЕТ.

Вредата (тежест на очакваната вреда) в измерителни единици (например левове загуба, дни на неработоспособност, левове за възстановяване) е с условни стойности за разглежданите случаи.

Възможно е да се извършат изчисления за риска при приети стойности и по двата метода, за всяка от структурите от фиг. 3 и фиг. 4 (които са съответно трансформирани от фиг. 1), чрез определяне вероятността за поява на:

- финалното събитие при FT;

- $\quad$ крайните събития (следствията) при ЕТ.

\section{Изчисляване на риска}

Анализът и оценката на риска е процес, при който се анализира риска за да може да се определи вероятността за реализация и възможните последствия от опасно събитие. Необходимо е да получим количествена оценка на всеки от рисковете така, че те да бъдат приоритизирани (например за целите на модифицирането на системите). Конкретният момент на настьпване на риска има значение върху последиците от неговото реализиране. Използвайки тези два показателя (вероятността и последствията) се въвежда т.нар. матрица за оценка на степента на риска [12].

При оценката на ефективността на технико-икономическите системи рискът се дефинира като продукт, получен от произведението на вероятността за претърпяване на вреда и тежестта на вредата, отнесено към определена времева единица [13] или изчисляването на риска се извършва чрез степента на риска, която се определя като математическо очакване на вредата от нежеланото събитие, т.е.:

$\mathrm{R}=\mathrm{P} . \mathrm{W}$,

където: P - вероятност за поява на събитието (например отказ на техническа система), $\mathrm{W}$ вреда, нежелани последствия.

\section{ЗАКЛЮЧЕНИЕ}

Разгледани са методите надеждностна блокова диаграма, дърво на отказите и дърво на събитията, с цел извършване на трансформация от една зададена структура до друга. Определянето на риска от техническа система, може да се извърши и чрез допълнителен междинен етап - трансформиране на структури, за да бъдат използвани предимствата на прилагания метод, като се избегнат съществуващите недостатъци.

Предложена е разработена методика за анализ на риска, като е показан пример за определяне на риска чрез трансформиране на структури приложим за обучение на студенти по анализ и управление на риска. Показана е приложимостта на методиката, от гледна точка на съответните предимства и недостатъци по двата метода. 


\section{АНАЛИЗ И ОЦЕНКА НА РИСКА ОТ РИСКОВИ ТЕХНИЧЕСКИ СИСТЕМИ ПРИ РАЗРАБОТВАНЕТО НА МЕТОДИКА ЗА ОБУЧЕНИЕТО НА СТУДЕНТИ ЧРЕЗ ТРАНСФОРМИРАНЕ НА СТРУКТУРИ}

\section{ЦВЕТЕЛИНА СИМЕОНОВА}

\section{ЛИТЕРАТУРА}

1. Христов Хр. Основи на осигурителната техника. София, изд. Техника, 1990. Hristov Hr. Osnovi na osiguritelnata tehnika. Sofiya, izd. Tehnika, 1990

2. Гиндев Е. Увод в теорията и практиката на надеждността - част 1 и 2: АИ "Проф. Марин Дринов". 2000, 2002. Gindev E. Uvod v teoriyata i praktikata na nadezhdnostta - chast 1 i 2: AI "Prof. Marin Drinov". 2000, 2002.

3. БДС EN IEC 61025 Анализ чрез дървото на отказите (FTA). BDS EN IES 61025 Analiz chrez darvoto na otkazite (FTA).

4. БДС EN 62502:2010 Методи за анализ на надеждността. Анализ чрез дървото на събитията (IEC 62502:2010). BDS EN 62502:2010 Metodi za analiz na nadezhdnostta. Analiz chrez darvoto na sabitiyata (IEC 62502:2010).

5. Clemens, P.L.; Rodney J. Simmons (March 1998). "System Safety and Risk Management". NIOSH Instructional Module, A guide for Engineering Educators. Cincinnati, OH: National Institute for Occupational Safety and Health: IX-3-IX-7.

6. Clemens. PL [1992]. Transformations, fault tree/reliability block diagram/event tree (lecture presentation). Tullahoma. TN: Sverdrup Technology. Inc. [Accessed on: 12 Nov. 2012]. (Viewed in: http://www.sverdrup.com/svt for presentation slides).

7. Clemens, PL [1993]. Fault tree analysis (lecture presentation). 4th ed. Tullahoma, TN: Sverdrup; Technology, Inc. [Accessed on: 12 Nov. 2012]. (Viewed in: http://www.sverdmp.com;svt for presentation slides).

8. Clemens, PL [1990]. Event tree analysis (lecture presentation).. 2nd ed. Tullahoma. TN: Sverdrup; Technology, Inc. [Accessed on: 12 Nov. 2012]. (Viewed in: http://www.sverdmp.com/svt for presentation slides).

9. Presentation slides. [Accessed on: 12 Nov. 2014]. Viewed in: http://www.sverdrup.com/svt for presentation slides that support this lesson.

10. Johnson Chris. Fault Trees and Software PRA [Accessed on: 14 Nov. 2018]. Viewed in: http://www.dcs.gla.ac.uk/ johnson/teaching/safety/powerpoint/8_FTA.pdf

11. Манчев Б. (Рис инженеринг ООД), Б. Маринов (Рис инженеринг ООД), н.с. І ст. Б. Ненкова (ИИ БАН). Приложение на метода "дърво на отказите" за анализ на системи. [Accessed on: 12 Nov. 2018]. Viewed in: https://inis.iaea.org/collection/NCLCollectionStore/_Public/32/007/32007930.pdf; Manchev B. (Ris inzhenering OOD), B. Marinov (Ris inzhenering OOD), n.s. I st. B. Nenkova (II - BAN). Prilozhenie na metoda "darvo na otkazite" za analiz na sistemi

12. Управление на риска. Уикипедия. [Accessed on: 11 Nov. 2018]. Viewed in: [https://bg.wikipedia.org/wiki/\%D0\%A3\%D0\%BF\%D1\%80\%D0\%B0\%D0\%B2\%D0\%BB\%D0\%B5\%D0\%BD\%D 0\%B8\%D0\%B5_\%D0\%BD\%D0\%B0_\%D1\%80\%D0\%B8\%D1\%81\%D0\%BA\%D0\%B0]. Upravlenie na riska. Uikipediya

13. Юридическият термин „риск“ в националната сигурност на Република България. [Accessed on: 11 Nov. 2018]. Viewed in: http://conf.uni-ruse.bg/bg/docs/cp15/7/7-24.pdf. YUridicheskiyat termin „risk“ v natsionalnata sigurnost na Republika Balgariya.

\section{Информация за автора:}

Ас. д-р инж. Цветелина Богданова Симеонова, София 1574, ул. Гео Милев 158, ВТУ „Т. Каблешков”, Тел.: 02 9709296, e-mail: ts.b.simeonova@abv.bg

\section{Contacts:}

Assist. professor Tsvetelina Simeonova PhD, T.Kableshkov University of Transport, 158 Geo Milev St., Sofia, office phone: +35929709296, e-mail: ts.b.simeonova@abv.bg

Дата на постъпване на ръкописа (Date of receipt of the manuscript): 05.01.2019.

Дата на приемане за публикуване (Date of adoption for publication): 05.03.2019. 